\title{
Spatial Information Transfer with a Stationary Coupling Wave in Rb Atoms
}

\author{
In Ho Bae and Han Seb Moon* \\ Department of Physics, Pusan National University, Geumjeong-Gu, Busan 609-735, Korea
}

(Received August 21, 2008 : revised September 15, 2008 : accepted September 16, 2008)

\begin{abstract}
We report on the spatial information of a coupling laser transfer into a reflected probe laser; the transfer is achieved by means of electromagnetically induced transparency through a common excited state connected with $5 \mathrm{~S}_{1 / 2}-5 \mathrm{P}_{1 / 2}\left(\mathrm{~F}=1 \rightarrow \mathrm{F}^{\prime}=2\right)$ in the ${ }^{87} \mathrm{Rb} \mathrm{D}_{1}$ line. When the coupling laser was spatially modulated as a stationary wave, the absorption of the probe laser was enhanced and the reflection of the probe laser was generated. When the coupling laser was spatially modulated by a mask, we observed that the reflection light of the probe laser was modulated as the shape of the mask. The Bragg reflection transferred the spatial information of the coupling laser. The reflection was approximately $7 \%$ of the incident power of the probe laser.
\end{abstract}

Keywords: spatial information; standing wave, Bragg reflection

OCIS codes : (300.6210) Spectroscopy, atomic; (300.6260) Spectroscopy, diode lasers; (300.6290) Spectroscopy, four-wave mixing

\section{INTRODUCTION}

Since the first observation of electromagnetically induced transparency (EIT) by Harris et al., EIT has become a subject of great academic interest phenomena for researchers of atomic coherent effects [1]. Early EIT studies on the multilevel system of atomic media with traveling coupling fields reported important results on the fundamental characteristics of EIT [2-4]. When light is transmitted in a resonant opaque medium by EIT, the group velocity of the light decelerates. An extreme case studied by Lukin and colleagues involves the storage of light [5].

EIT is strongly related to the absorptive and dispersive property of light in a resonant medium. One of the earlier reports said EIT was achieved in an absorbing medium driven by stationary coupling fields. When the stationary coupling fields periodically modulate the absorptive property of the medium, the transmittance of EIT is reduced and the reflection light is generated by periodic modulation of the absorptive property in the $\mathrm{Rb}$ vapor cell; this latter process is similar to an electromagnetically induced grating (EIG) [6-11].

In the three-level $\lambda$-type system, Ling et al. studied the diffraction of a probe field by EIG in a homogeneous atomic system [6]. Imoto et al. observed the experimental results of EIG in the three-level system of cold sodium atoms [7]. Xiao et al. researched the transmission spectrum of EIG with the generation of diffraction light in a pure $\mathrm{Rb}$ vapor cell. For the absorption of an EIG transmission spectrum in a pure $\mathrm{Rb}$ vapor cell, EIG alters the electromagnetically induced absorption grating (EIAG) [8]. Hemmar et al. presented experimental results on diffraction light, which is phase-conjugate (PC) light; their process involved four-wave mixing in a hot sodium cell with 5 mTorr of a helium buffer gas [9].

In this paper, we show a spectral profile of the transmission and reflection spectrum of EIAG in a $5 \mathrm{~S}_{1 / 2}-5 \mathrm{P}_{1 / 2}$ $\lambda$-type system of ${ }^{87} \mathrm{Rb}$ and the spatial information transfer of a spatially modulated coupling field by means of a mask. When the spatial shape of the coupling (Lc) laser was modulated by the mask, the shape of the probe (Lp) laser is reflected by the stationary coupling field. The phase-conjugation light for our system is the reflected probe laser by the periodical modulation of the coupling (Lc) laser in the interactive region. The spatial modulation transfer is studied for the masks in the shape of a cross and the letter G.

*Corresponding author: hsmoon@pusan.ac.kr 


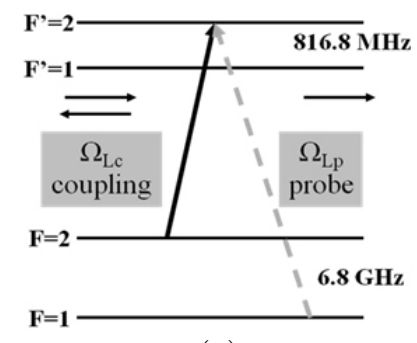

(a)

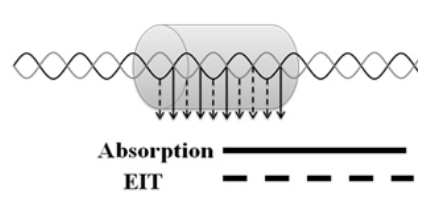

(b)
FIG. 1. (a) Schematic energy system of EIAG experiments in a $5 \mathrm{~S}_{1 / 2}-5 \mathrm{P}_{1 / 2} \lambda$-type system of ${ }^{87} \mathrm{Rb}$; (b) periodic modulation of the absorption of the Lp laser between the nodes (absorption; solid line) and the anti-nodes (EIT; dashed line) in an $\mathrm{Rb}$ vapor cell.

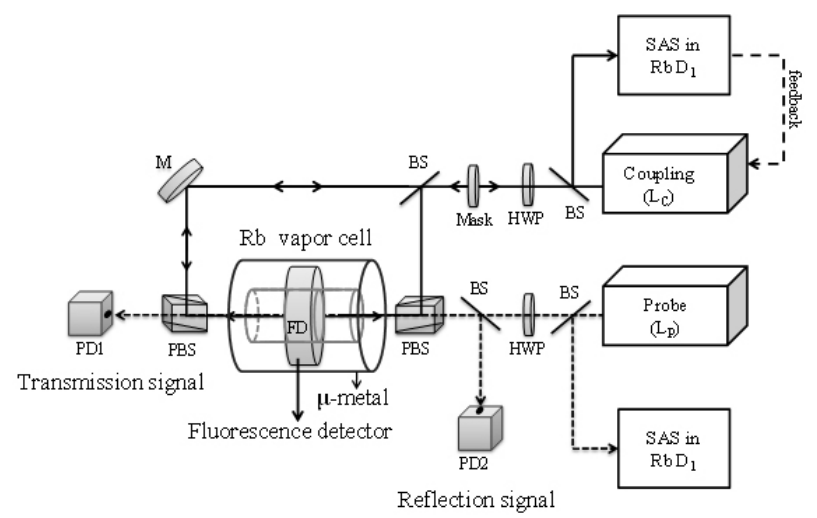

FIG. 2. Full experimental setup of the EIAG in a $5 \mathrm{~S}_{1 / 2^{-}}$ $5 \mathrm{P}_{1 / 2} \lambda$-type system of ${ }^{87} \mathrm{Rb}$ (BS: beam splitter; PBS: polarization beam splitter; PD: photo diode; HWP: half-wave plate; and FD: fluorescence detector).

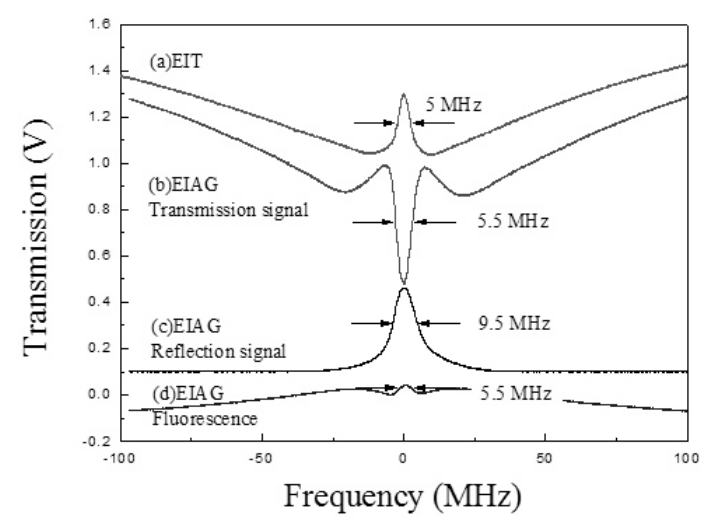

FIG. 3. (a) A typical EIT in a $5 \mathrm{~S}_{1 / 2}-5 \mathrm{P}_{1 / 2} \lambda$-type system of ${ }^{87} \mathrm{Rb}$; (b) the transmission signal of EIAG; (c) the reflection signal of the Lp laser by EIAG; and (d) the fluorescence inside the ${ }^{87} \mathrm{Rb}$ vapor cell.

\section{EXPERIMENTAL SETUP}

Figure 1(a) shows a schematic energy system for the EIAG. The splitting frequency of excited states of the $\mathrm{D}_{1}$ line of the ${ }^{87} \mathrm{Rb}$ atom is $816.8 \mathrm{MHz}$ and one of the ground states is $6.8 \mathrm{GHz}$. The laser fields, which are resonant with two ground states and connected though a common excited state corresponding to one of the hyperfine states of the $5 \mathrm{P}_{1 / 2}$, form the $\lambda$ scheme. The stationary coupling field (Lc) is composed of two waves traveling in opposite propagating directions; the two waves are generated from the one laser system. As shown in Fig. 1(b), the absorption of the probe field is periodically modulated at the nodes and anti-nodes in the $\mathrm{Rb}$ vapor cell with the stationary coupling field. The solid lines and the dashed lines in Fig. 1(b) indicate the linear absorption and the EIT, respectively. When the amplitude of the stationary coupling field is periodically changed between the nodes and anti-nodes, the absorption of the probe field is modulated as a result of the linear absorption at the nodes and the EIT at the anti-nodes. Under this periodical modulation of the absorption of the probe field, a $1 \mathrm{D}$ photonic bandgap is generated and a part of the probe field is reflected by an absorptive Bragg grating [10].

The experimental setup for the EIAG in a $5 \mathrm{~S}_{1 / 2}-5 \mathrm{P}_{1 / 2}$ $\lambda$-type system of ${ }^{87} \mathrm{Rb}$ is shown in Fig. 2 . For our experiment, we used two independent external cavity diode lasers of the grating feedback type. The Lc laser and the $\mathrm{Lp}$ laser are resonant for a $\mathrm{F}=2 \rightarrow \mathrm{F}^{\prime}=2$ transition and the $\mathrm{F}=1 \rightarrow \mathrm{F}^{\prime}=2$ transition in the ${ }^{87} \mathrm{Rb} \mathrm{D}_{1}$ line, respectively. The frequencies of the two independent diode lasers are fixed by means of saturated absorption spectroscopy. The $\mathrm{Rb}$ vapor cell has a diameter of 2.5 $\mathrm{cm}$ and a length of $5 \mathrm{~cm}$. The vapor cell was thermally stabilized through heating tapes at a temperature of 90 ${ }^{\circ} \mathrm{C}$. To prevent interference from the Earth's magnetic fields, we wrapped the cell in $\mu$-metal. The polarizations of the Lc and the Lp lasers were linear and perpendicular to each other. The two lasers were overlapped and controlled with a polarization beam splitter (PBS) and a half-wave plate in the beam path. After passing the cell, the Lc and the Lp lasers were separated by means of the PBS to detect the pure Lp laser signal. To transfer the spatial information of the Lc laser into the reflected Lp laser, we placed a mask in the propagating path of the Lc laser and changed the spatial profile of the Lp laser with the mask. When the reflection light of the Lp laser was generated by EIAG, the reflection light of the probe laser could be modulated in a manner similar to the shape of the mask.

\section{EXPERIMENTAL RESULTS}

The spectrum of Fig. 3(a) represents the EIT in the $5 \mathrm{~S}_{1 / 2}-5 \mathrm{P}_{1 / 2} \lambda$-type system of ${ }^{87} \mathrm{Rb}$, where the $\mathrm{Lp}$ laser power was $16 \mu \mathrm{W}$ and the Lc laser power was 13.7 $\mathrm{mW}$. When the Lc laser is changed from the traveling fields to the stationary fields, the transmission of EIT alters the EIAG signal, as shown in Fig. 3(b). When 


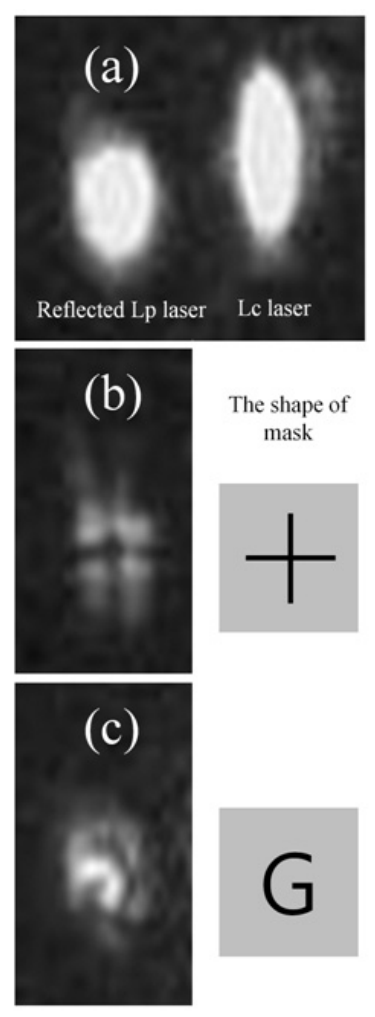

FIG. 4. Images of the Lc laser and the reflected Lp laser in the far field: (a) without a mask, the original spatial shapes of the Lc laser (right) and the reflected Lp laser (left) generated by EIAG; (b) the spatial shape of the reflected Lp laser with the cross-shaped mask $(+)$; and (c) the spatial shape of the reflected Lp laser with letter G-shaped mask.

the absorption peak resembles that of the reversed-EIT of EIAG, the Lp laser enhances the two-photon resonance and acts as an alternative for the two traveling Lc fields after the passage from the nodes of the coupling fields [11]. When the coupling laser was spatially modulated as a stationary wave, the reflection of the probe laser was generated by the 1-D photonic bandgap and then detected by the photodiode 2 (PD2). The spectrum of Fig. 3(c) shows the reflection light of the Lp laser by EIAG. The power of the reflection light was only $7 \%$ of the incident power of the Lp laser. The linewidth of the reflected Lp was broader than that of the transmission spectrum of either the EIT or EIAG because the grating is induced by absorptive property [12]. Even though only $7 \%$ of the Lp laser was reflected, we thought that most parts of the Lp laser were absorbed by EIAG. The spectrum of Fig. 3(d) shows the simultaneously measured fluorescence spectrum inside the $\mathrm{Rb}$ vapor cell. The peak of the fluorescence spectrum represents the enhanced absorption by EIAG. If there were no absorption of the Lp laser by EIAG, there would be no fluorescence peak but only a reduction of the transmission peak of the EIT.
Figure 4 shows spatial images of the reflected Lp laser by EIAG, where the Lp laser power was $1 \mathrm{~mW}$ and the Lc laser power was $13.7 \mathrm{~mW}$. The image of Fig. 4(a) displays the original spatial shape of the Lc laser without the mask and the reflected Lp laser generated by EIAG. The shape of the Lc laser is shown on the right-hand side of Fig. 4(a), and the reflected Lp laser is shown on the left-hand side of Fig. 4(a). The reflection light is circular. As shown in Figs. 4 (b) and 4(c), when the spatial shape of the Lc laser was changed by the mask, the shape of the reflected Lp laser was also changed.

To investigate the spatial information transfer of the Lc laser in relation to the shape of the mask, we made two masks: one shaped like a cross $(+)$ and the other shaped like the letter $\mathrm{G}$. The real masks are shown in right-hand side of figure 4(b) and 4(c). Figure 4(b) shows the spatial shape of the reflected Lp laser with the Lc laser spatially modulated by the cross-shaped mask. The coupling beam is screened by the used mask and there is no coupling beam in the shape of the mask. We observed that the dark shape of the reflected Lp laser is the same as the shape of the mask. Because there is no interaction between the Lp laser and the Lc laser, the dark region is similar to the font of the mask of the Lc laser.

The letter G shape of Fig. 4(c) is made by a mask equal to the dark shape. The shape of the mask (the G shape) was modified on the left-hand side and righthand side of the image of the reflection light. The bilateral symmetry occurs because the reflection light is the PC light, which has opposite propagating direction to original propagating direction of the Lp laser. Consequently, the images are overlapped in the Fig. 4 by folding both reflected Lp laser and masks. Although the reflection light is the part of the Lp laser, the reflected light of the Lp laser as shown Figs. 4(b) and 4(c) represents the spatial shape of the Lc laser as modulated by the mask. From these results, we can confirm that the spatial information of the Lc laser was transferred into the reflection light of the Lp laser.

\section{CONCLUSION}

We transferred the spatial information of stationary coupling fields into the reflection light of a probe laser delivering a Doppler-broadened $\lambda$-type atomic system. The absorption of the transmittance of the probe laser was enhanced, and the reflection light of the probe laser was generated in the stationary coupling fields. The spatial information of the coupling laser can be transferred into the reflected probe laser by the EIT in the ${ }^{87} \mathrm{Rb}$ $\mathrm{D}_{1}$ line. The reflection of the probe laser transferred the spatial information of the coupling laser. When the spatial information of the coupling laser was modulated by a mask, the spatial information of the reflection light 
of the probe laser was also modulated in a manner similar to the shape of the mask. Consequently, the spatial information of the Lc laser can be transferred to the shape of the reflection light of the Lp laser by a coherent atomic effect caused by stationary coupling fields. In the next experiment, we plan to upgrade the resolution of the spatial information transfer by means of phase lithography and store the spatial information after the light storage experiment.

\section{ACKNOWLEDGMENT}

This work was supported by Ministry of Education \& Science Technology through the Project of "Development of advanced measurement technology for public safety” (KRISS-08-2402-221). It was also supported by the Korea Science and Engineering Foundation(KOSEF) grant funded by the Korea government(MOST) (No. R01-2007-000-11636-0).

\section{REFERENCES}

[1] K. J. Boller, A. Imamoglu, and S. E. Harris, "Observation of electromagnetically induced transparency," Phys. Rev. Lett., vol. 66, no. 20, pp. 2593-2596, 1991.

[2] S. E. Harris, J. E. Field, and A. Kasapi, "Dispersive properties of electromagnetically induced transparency," Phys. Rev. A, vol. 46, no. 1, R29-R32, 1992.

[3] M. Xiao, Y. Li, S. Jin, and J. Gea-Banacloche, "Measurement of dispersive properties of electromagnetically induced transparency in rubidium atoms," Phys. Rev. Lett., vol. 74, no. 5, pp. 666-669, 1995.
[4] H. S. Moon, H. A. Kim, B. S. Kim, and J. B. Kim, "Electromagnetically induced transparency in an ideal three- level system in 87Rb atoms," J. Korean Phys. Soc., vol. 35, no. 3, pp. 207-211, 1999.

[5] D. F. Phillips, A. Fleischauer, A. Mair, R. L. Walsworth, and M. D. Lukin, "Storage of light in atomic vapor," Phys. Rev. Lett., vol. 86, no. 5, pp. 738-786, 2001.

[6] H. Y. Ling, Y. Q. Li, and M. Xiao, "Electromagnetically induced grating: Homogeneously broadened medium," Phys. Rev. A, vol. 57, no, 2, pp. 1338-1344, 1998.

[7] M. Mitsunaga and N. Imoto, "Observation of an electromagnetically induced grating in cold sodium atoms," Phys. Rev. A, vol. 59, no. 6, pp. 4773-4776, 1999.

[8] A. W. Brown and M. Xiao, "All-optical switching and routing based on an electromagnetically induced absorption grating," Opt. Lett., vol. 30, no. 7, pp. 699-701, 2005.

[9] P. R. Hemmer, D. P. Katz, J. Donoghue, M. CroninGalomb, M. S. Shahriar, and P. Kumar, "Efficient lowintensity optical phase conjugation based on coherent population trapping in sodium," Opt. Lett., vol. 20, no. 9, pp. 982-984, 1995.

[10] X. M. Su and B. S. Ham, "Dynamic control of the photonic band gap using quantum coherence," Phys. Rev. A, vol. 71, pp. 013821, 2005.

[11] F. Silva, J. Mompart, V. Ahufinger, and R. Corbalan, "Electromagnetically induced transparency in Dopplerbroadened three-level systems with resonant standingwave drive," Europhys. Lett., vol. 51, no. 3, pp. 286292, 2000.

[12] M. Bajcsy, A. S. Zibrov, and M. D. Lukin, "Stationary pulses of light in an atomic medium," Nature, vol. 426, pp. 638-641, 2003.K. J. Boller, A. Imamoglu and S. E. Harris, "Observation of electromagnetically induced transparency," Phys. Rev. Lett., vol. 66, no. 20, pp. 2593-2596, 1991. 